\title{
Efficiency of nanoparticle reinforcement using Finite Element Analysis of Titanium alloy mandible plate
}

Prashant Jindal $^{\mathrm{a}}$, Frank Worcester ${ }^{\mathrm{b}}$, Anand Gupta ${ }^{\mathrm{c}}$, Philip Breedon ${ }^{\mathrm{b} *}$

${ }^{a}$ University Institute of Engineering \& Technology, Panjab University, Chandigarh 160

014, India $;{ }^{b}$ Medical Design Research Group, Nottingham Trent University,

Nottingham, UK; ${ }^{\circ}$ Department of Dentistry, Government Medical College and Hospital,

Sector 32, Chandigarh-160030, India.

\section{* Corresponding Author:}

Professor Philip Breedon,

Director, Medical Design Research Group,

Nottingham Trent University, Nottingham, UK

+44 11584 86424; philip.breedon@ntu.ac.uk
Abbreviations:
(NPs) Nanoplatlets
(NTs) Nanotubes 
Nanoparticles in the form nanotubes and nanoplatlets have been compared for Von Mises stresses by using them as low composition reinforcements in Titanium alloy based mandible plate for different compositions and orientations. A Finite Element Model (FEM) has been designed to reconstruct a fractured human mandible with a Titanium alloy mandible plate. A 500N compressive force was applied on the mandible and stress distribution across the plate sections was analysed for aligned 2D random and 3D random orientations for both tubes and platlets. Carbon material as graphene has been used for tube and platlet in the form of Nanotubes (NTs) and Nanoplatlets (NPs) respectively. Using properties of graphene as the filler in Titanium alloy plate, for both NPs and NTs, the stresses reduced between 5\% to 25\% for NPs and NTs Graphene-Titanium composite plates in comparison to pure plates, at critically stressed sections. NTs exhibited stress reduction of nearly $23.4 \%$ for aligned configurations while NPs exhibited stress reduction up to $21.2 \%$ for $2 \mathrm{D}$ and $3 \mathrm{D}$ random configurations in comparison to pure Titanium plates. Hence, it has been suggested that nanotubes exhibited superior mechanical reinforcement potential beyond that of nanoplatelets for aligned, while nanoplatelets provided enhanced mechanical reinforcements for random configurations. Therefore, for biomedical implant applications nanocomposite materials can be designed with the same dimensional form but with lower compositions of filler materials by simply manipulating the appropriate orientations.

Keywords: Finite Element Modelling/ Analysis [Medical], Implants/ Prosthetics, Mathematical Modelling [Medical], Modelling/ Simulation [Biomechanics], Stress Analysis/ Testing [Biomechanics]

\section{Introduction}

Biomedical implant materials based on metal alloys, are always desired to be light in weight, small in size, biocompatible and have the appropriate mechanical properties. 
With the advent of carbon based nanoparticles including nanospheroidals, nanoplatlets and nanotubes, there has been growing interest in designing new composite materials for different types of engineering and biomedical applications. The addition of these nanoparticles into polymer/metal matrix materials has been observed to significantly enhance the mechanical properties of the host matrix, promising a novel class of nano composite materials with superior properties and added functionalities [1]. Biomedical implants [2,3] made up of different types of metal alloys are being widely used for orthopaedic, dental, maxillofacial and craniofacial reconstruction applications. Carbon materials [4-6] in the form of Carbon Nanotubes (CNTs) and Graphene have shown tremendous potential for enhancing mechanical properties of polymer $[7,8]$ and metal [9] composite materials without altering their mass significantly. Graphene can be used experimentally in multiple forms [4] such as microplates, nanoplates, oxides, and nanosheets. On a bulk level, Graphene [10-12] has a thermal conductivity of $3000 \mathrm{~W} / \mathrm{mK}$, elastic modulus nearly $1 \mathrm{TPa}$, ultimate tensile strength $130 \mathrm{GPa}$, shear modulus of 53GPa, Poisson's ratio of 0.19 and mass density of nearly $700 \mathrm{~kg} / \mathrm{m}^{3}$. These major properties of graphene play an important role for enhancement of mechanical properties of any composite material. All these properties of graphene are dependent upon chirality, layer thickness, orientation, direction of loading and forms, hence lot of variations [13-15] based on these parameters have also been reported. Graphene and its various forms have shown biocompatibility [16] for different applications like drug delivery, tissue engineering, bio sensing and implants. The ability of graphene to improve the mechanical and biological [17] properties of implants or scaffold materials, by promoting adhesion, proliferation, and osteogenic differentiation have also been demonstrated in several studies. Graphene coated nitinol [18] has been proposed as a viable candidate for stents, however, numerous challenges remain due to exogenous 
material cytotoxicity, bio- and hemo-compatibility.

Among the bio-medical implants, Titanium alloy(Ti-6Al-4V) [2,19] based maxillofacial and mandible plates are widely used for jaw fracture treatment and reconstructions. Based on the method of manufacturing of these Titanium alloy plates, their elastic moduli and fatigue strength vary between $110-114 \mathrm{GPa}$ and $600-816 \mathrm{MPa}$ respectively. Plates of variable thickness and lengths are used, based on the type of fractures. Plates need to be evaluated for strength under various jaw movement conditions for which various FEM techniques are adopted. FEM is also used to design different shapes, combinations and thickness of these plates to evaluate their effects on strength enhancement for withstanding higher mandible stresses. Gutwald et al [20] evaluated customized mandibular reconstruction plate strengths using mechanical testing and FEM. He reported that maximum stress was significantly reduced by nearly $31 \%$ by increasing the bar width from $5.5 \mathrm{~mm}$ to $6.5 \mathrm{~mm}$. Goulart et al [21] used FEM by applying von Mises yielding criteria for evaluating the effectiveness of using 2 plates instead of single for recovery of bone fractures and observed that two locking plates promoted a better mechanical resistance for complex mandible fractures. ANSYS software has also been used for FEM analysis by applying von Mises yielding criteria to evaluate mechanical stresses[22] in the plate by simulating masticatory forces in the human jaw to provide stress intensifiers and breaking points on the plate.

These methods can also assist in designing customized and individualized plates of different geometry and shapes. Improvised late designs [23] for minimising stresses in plate and screws were reported by evaluating stresses in mandible bone for different plate designs.

Research undertaken by various scientists clearly indicates an increasing interest in improving the mechanical strength of biomedical implants. FEM techniques have 
been widely used for characterisation to save on manufacturing costs. Various software, failure theories, plate types and geometries have been preferred to explore these properties and accordingly produced satisfactory results in several cases. Along with the plate designs, FEM can also be used to evaluate the influence of a composite filler material on the mechanical properties of plates. Graphene, despite of being a mechanically strong material, has not been exhaustively explored for maxillofacial and mandible plate applications. To optimize the use of any such promising filler particle, it is imperative to evaluate the type of suitable nano-particle (NP or NT) and its orientation in the base matrix. Many different nanoparticle and host combinations have been investigated [24-26]. However, a systematic examination of the reinforcing effect of nanoparticles in the same matrix have not been reported exhaustively.

For this paper, a basic Titanium alloy based thin mandible plate for a mandible fractured model, with a minor quantity of Carbon based NPs and NTs with physical properties of Graphene has been modelled and analysed for von mises stress distribution using SolidWorks software. The influence of NPs and NTs for different orientations (aligned, 2D random and 3D random) in the plate has been analysed and compared with non-reinforced plate under the application of $500 \mathrm{~N}$ compressive force on the mandible which is equivalent to bite/chewing forces[20,27] experienced within the human jaw, so that any improvement in the plate design in terms of stress relief at critical sections could be explored. Reduction in stress at plate sections, could lead to an improvised design by altering the size or weight of the plate.

\section{Materials and Methods}

\subsection{Finite Element Model}

A mandible bone model with a $1 \mathrm{~mm}$ fracture was used and a Titanium alloy(Ti-6Al-4V) 
based $1 \mathrm{~mm}$ thick plate was used for its fracture osteosynthesis. As shown in Figure 1, the plate was placed at the suitable location for fracture osteosynthesis and a compressive bite force $[20,27] 500 \mathrm{~N}$ was applied at the mandible with fixed supports at the opposing end. Half part of the mandible has been considered as a representation for the complete strucuture. Based on similar conditions of loading and fixtures, the other symmetrical half mandible is also expected to behave similarly. Moreover, the primary focus of this study is to only evaluate effect of NPs and NTs inside the plate due to mandible movement under bite forces, which would be identical for other symmetrical mandible structure also.

The Titanium plate was modelled as shown in Figure 2 with customized screws represented by a cylinder ( $7 \mathrm{~mm}$ length) and a rigidly connected head ( $2.6 \mathrm{~mm}$ diameter) to the bone and plate respectively [28]. Four unicortical screws were used with plate of thickness $1 \mathrm{~mm}$, length $16.5 \mathrm{~mm}$, diameter $3 \mathrm{~mm}$ and volume $22.02 \mathrm{~mm}^{3}$. The plate and screws were Ti-6Al-4V alloy based, hence their mechanical properties were considered as- Elastic modulus $105 \mathrm{GPa}$, Poisson ratio 0.31, yield strength $827 \mathrm{MPa}$ and mass density $4429 \mathrm{~kg} / \mathrm{m}^{3}$.

Considering the basic structure of NPs and NTs as shown in Figure 3, their orientations inside the plate were varied. Liui et al [1] have reported the different orientations for NPs and NTs as aligned, 2D and 3D random.

Random 2D configurations were obtained by rotating the reinforcements in steps of $45^{\circ}$ along one axis for both NTs and NPs. Random 3Dconfigurations were obtained by rotating $45^{0}$ along two axes, thereby creating a random orientation along two planes, as shown in Figure 4.

Based on this model, other plate models were designed with both reinforcements aligned(Figure 5(a-c)), 2D and 3D randomly(Figure 6(a-c)) oriented [1] and embedded 
on the at the plate centre near the exact fracture location, as this was the expected breaking [20] and most vulnerable section of the plate during mandible movement. Figure 5(a-c) show the model arrangement with different NP configurations while Figure 6(a-c) show similar arrangements for NT configurations.

To evaluate the effect of angular orientation of both types of nanoparticles in the plate, dimensions were taken as shown in Figure 3 for both geometries in the form of a bunch of NP and NTs. An equivalent bulk density [4] of $700 \mathrm{~kg} / \mathrm{m}^{3}$, Young's modulus 1TPa and Poisson's ratio 0.2 was defined for both the nanoparticles. Mandible bone elastic modulus was taken as $18 \mathrm{GPa}$, Poisson's ratio 0.394 and mass density $1.8 \mathrm{gm} / \mathrm{cm}^{3}$. Based on the densities and volumes, the given mass of the plate was $97.4 \mathrm{mg}$, NPs weighed nearly $0.002 \mathrm{mg}$ and NTs weighed nearly $0.001 \mathrm{mg}$ which is an insignificant increment to the overall weight of the plate. The bulk density for the filler could vary up to $2000 \mathrm{~kg} / \mathrm{m}^{3}$ which would result in its weighing mass up to $0.003 \mathrm{mg}$ and $0.006 \mathrm{mg}$ for NPs and NTs respectively, which would still remain insignificant to the overall weight of the plate. Dimensions of NPs and NTs vary between 0.1 to $100 \mu \mathrm{m}(1)$ however, for such smaller geometries, the quantitiy of filler material used for fabricating or modelling the reinforced plate would be less than $0.1 \mu$ grams. Therefore, in this paper, bunches of fillers have been taken to facilitate modelling and futuristic handling and manufacturing procedures. Also, despite of increased filler dimensions, the overall mass of fillers does not exceed $6 \mu$ grams $(0.002 \mathrm{wt} \%$ of plate $)$ which is insignificant.

Fixed compressive force of 500N was applied on these configurations and various other angular $\left(135^{\circ}, 90^{\circ}\right)$ orientations for both NPs and NTs filler compositions to evaluate stress at the critical point on the plates.

\subsection{Finite Element Analysis}

A non-reinforced Titanium Alloy plate model was a high quality curvaturebased 
mesh for a quadratric 10 node tetrahedron element to obtain the most accuratre results. There were a total of 598968 degrees of freedom, 1634436 total number of elements and 2286804 nodes. A static simulation study under compression loading was conducted on the mandible with specified $(500 \mathrm{~N})$ force equivalent to biting forces. Figures 7(a)-(b) indicate the maximum stress elements near the middle section and upper surface for the non-reinforced plate adjacent to the actual fracture location.

To compare the effects of various orientations of NTs and NPs, same simulation studies under finer mesh as shown in Figures 8(a)-(b) of composite mandible plates were repeated and analysed for stress distributions across at same sections as nonreinforced plate.

Figures 9(a)-(b) indicate stress distributions for NT aligned configurations while Figure 10 indicates stress distributions for NP 2D random configuration. These figures demonstrate that the stresses were significantly reduced in the same sections of the plate which were identified as vulnerable to failure.

\section{Results}

Finite Element Analysis (FEA) results of various models are shown in Figure 11 and Table 1. It can be observed that presence of NPs and NTs successfully reduced the stress distribution across the vulnerable sections

For the non-reinforced plates, critical stress points were spread along the middle section of the plate indicating the vulnerable breaking sections. Stresses across the section, were averaged out and compared with the same sections for NT and NP configurations. For aligned configurations, NT reinforced plates showed nearly $23.4 \%$ lower stress distribution along the critical sections in comparison to the non-reinforced plates. Aligned NP configurations also showed stress reduction by $19.5 \%$ across the 
same sections in comparison to non-reinforced plates. For models designed for 2D random orientations, NP reinforced plates showed improvements in stress reduction in comparison to both non-reinforced and NT reinforced plates. Similar reduced trend for 3D randomly oriented NP plates was also observed.

Both NP and NT fillers differ on aspect ratio and orientation, hence, effective surface area $\left(S_{N P, N T}\right)$ per unit particle volume $\left(V_{N P, N T}\right)$ [1] for each of them becomes essential as shown in $\mathrm{Eq}(1)$ and $\mathrm{Eq}(2)$

$$
\begin{aligned}
& \beta_{N P}=\frac{S_{N P}}{V_{N P}}=\frac{\frac{\pi d^{2}}{2}+\pi d t}{\frac{\pi d^{2} t}{4}} \\
& \beta_{N T}=\frac{S_{N T}}{V_{N T}}=\frac{\frac{\pi t^{2}}{2}+\pi d t}{\frac{\pi t^{2} d}{4}}
\end{aligned}
$$

Here $d$ is the diameter and $t$ represents thickness for NP in $\mathrm{Eq}(1)$ while $t$ represents diameter and $d$ is the thickness for NT in $\mathrm{Eq}(2)$. Moreover, the dimensional values for diameter of NP is same as length of NT and thickness of NP is same as diameter of NT. Hence the comparison for both configurations is given in $\mathrm{Eq}(3)$

$$
\frac{\beta_{N T}}{\beta_{N P}}=\frac{T+2 d}{d+2 t}=\frac{5}{4}
$$

This ratio is primarily dependent upon the aspect ratio of both the particles and if $d>>t$ then this ratio may vary between 1.25 to 2.0 . Hence, it indicates that the NT configurations can have a stronger interaction with the matrix and therefore may result in a superior reinforcement than NP.

\section{Discussion}

For the same aspect ratio for longitudinal properties of aligned Titanium alloy composites, the mechanical reinforcing efficiency of NTs is higher than that of NPs, while the geometrical features of NPs allow better reinforcement for most cases of 
random orientation and higher aspect ratio nanoparticles. Both reinforcements provide significant stress reduction across the plate sections, owing primarily to the superior mechanical properties of Graphene. These Graphene NP and NT fillers, absorb majority of the stress distribution, thereby, relieving the actual plate from breakage significantly. A reduction of stress in a range of $21 \%$ to $23 \%$, without significant alteration in the overall mass of the plate is an important finding. NTs $(0.001 \mathrm{wt} \%)$ contribution to the total weight of the plate was nearly half in comparison to NPs $(0.002 \mathrm{wt} \%)$, still for aligned configurations they exhibited superior stress reduction in the plate. It has been suggested that at the same volume fraction, identical degree of dispersion and extent of influence on the surrounding molecules, NTs could generate a significantly larger amount of interphase than NPs. This difference could effect a significant change in the bulk performance of the nanocomposites, depending on the nature of nanoparticle-metal material interaction. This may also explain the reason for lower improvements in properties among graphite nanoparticles (29) in comparison to carbon nanotube reinforced samples (30). However, for the random orientations of these composites, it has been suggested that, the amount of interphase, aspect ratio and surface to volume ratios are dominated by the weight composition of NP in comparison to NT. Since, the minor weight composition of NP is approximately twice that of NT, accordingly the mechanical strength of graphene contributes significantly. With reports on successful biocompatible applications of Graphene on biosensors, drug delivery and tissue engineering, it could also be used as a suitable filler material for improving mechanical properties of biomedical plates. Random dispersion of any filler is most effective in enhancing mechanical strength $(31,32)$ especially when its related to nano-composites. Hence, controlling the orientations (33) has always been a prime focus in order to save on material cost. During manufacturing, it requires additional methods to control the 
orientation of fillers and fibres and their effectiveness can only be explored post manufacturing, therefore finite element studies can provide important data for the relative research community making decisions related to manufacturing. Models have indicated that based on the dexterity to manipulate orientation, choice of reinforcement as NP or NT can be made for producing similar stress reduction results in a metal plate.

Many complexities for these models and composites have been excluded, however, this simpler FEM approach provides an insight and generalized guidance in characterizing and designing of nanocomposites using nanotubes or nanoplatelets as reinforcing nanoparticles. These results motivate and provide a basis for building a consensus on mechanical characterization of different nanoparticle based composite materials. Further studies could be undertaken based on these geometrical arrangements by varying the dispersion, quantity, distribution, extent of inter phase zone and comparing the results with different modelling, simulation and experimental studies.

\section{Conclusions}

Nanocomposite fabrication methods incur large manufacturing costs, and hence it becomes imperative to analyse the nanocomposite design by modelling and simulation studies. For this paper, a Titanium alloy based mandible plate was designed and reinforced as a composite plate with different configurations (aligned, 2D and 3D random) of graphene based nanoparticles (NTs and NPs) under a compressive load of 500N(human bite force). Presence of both types of nano particles successfully reduced the stress distribution between $5 \%$ to $25 \%$ at critical sections of the titanium plate. Random configurations for NPs reduced the stress distribution more effectively in comparison to NTs while for aligned configurations, NTs could reduce the stress distribution more effectively. It has been suggested that due to greater weight of NPs $(0.002 \mathrm{wt} \%)$ than NTs $(0.001 \mathrm{wt} \%)$, the effect of Graphene as the filler contributed favourably in the stress distribution for random orientations. However, for the aligned configurations NTs could have generated a significantly larger amount of 
interphase zone than NPs due to greater surface to volume ratio. Maximum stress reduction for both the reinforcements under different configurations was nearly $24 \%$ which suggests Titanium alloy mandible plates can be designed for higher mechanical strength by embedding minor compositions of nano-graphene nanoparticles without any variation in the overall weight of the plate.

Ultimately this work provides an insight at a broader level, stating that a biocompatible, mechanically strong, lightweight biomedical plate with a smaller form factor could be designed using the proposed nanoparticles, providing benefits to both patients and surgeons. For future work, optimal quantity and distribution of nanoparticles across the plates and their effects on mandible bone could also be explored.

\section{Acknowledgments}

Funding: Dr. Prashant Jindal is currently working as Commonwealth Rutherford Fellow(CSC ID: INRF-2017-146) within the Medical Design Research Group at Nottingham Trent University, Nottingham, UK and gratefully acknowledges the Commonwealth Scholarship Commission in the UK for their support.

\section{References}

1. Liu H, Brinson LC. Reinforcing efficiency of nanoparticles: A simple comparison for polymer nanocomposites. Compos Sci Technol [Internet]. 2008;68(6):1502-12. Available from: doi:10.11138/orl/2016.9.1S.107.

2. Pacifici L, De Angelis F, Orefici A, Cielo A. Metals used in maxillofacial surgery. ORAL Implantol. 2016;9(Table 2):107-11.

3. Azevedo CRF, Hippert E. Failure analysis of surgical implants in Brazil. Eng Fail Anal. 2002;9(6):621-33.

4. Papageorgiou DG, Kinloch IA, Young RJ. Mechanical properties of graphene 
and graphene-based nanocomposites. Prog Mater Sci [Internet]. The Authors; 2017;90:75-127. Available from: http://dx.doi.org/10.1016/j.pmatsci.2017.07.004 5. Jindal P, Goyal M, Kumar N. Mechanical characterization of multiwalled carbon nanotubes-polycarbonate composites. Mater Des [Internet]. Elsevier Ltd; 2014 Feb [cited 2013 Oct 24];54:864-8. Available from: 10.1016/j.matdes.2013.08.100 6. Mathur RB, Chatterjee S, Singh BP. Growth of carbon nanotubes on carbon fibre substrates to produce hybrid/phenolic composites with improved mechanical properties. Compos Sci Technol [Internet]. 2008 Jun [cited 2013 Oct 25];68(7-8):1608 15. Available from: 10.1016/j.compscitech.2008.02.020

7. Mathur RB, Pande S, Singh BP, Dhami TL. Electrical and Mechanical Properties of Multi-Walled Carbon Nanotubes Reinforced PMMA and PS Composites. Polym Compos [Internet]. 2008;29(7):717-27. Available from: https://doi.org/10.1002/pc.20449

8. Jindal P, Sain M, Kumar N. Mechanical characterization of PMMA / MWCNT composites under static and dynamic loading conditions. Mater Today Proc [Internet]. Elsevier Ltd.; 2015;2(4-5):1364-72. Available from: http://dx.doi.org/10.1016/j.matpr.2015.07.055

9. Bakshi SR, Lahiri D, Agarwal A. Carbon nanotube reinforced metal matrix composites - a review. Int Mater Rev [Internet]. 2010;55(1):41-64. Available from: 10.1179/095066009X12572530170543

10. Potenza M, Cataldo A, Bovesecchi G, Corasaniti S, Coppa P, Bellucci S. Graphene nanoplatelets: Thermal diffusivity and thermal conductivity by the flash method. AIP Adv. 2017;7(7).

11. Liu X, Metcalf TH, Robinson JT, Perkins FK, Houston BH. Internal Friction and 
Shear Modulus of Graphene Films. In: Internal Friction and Mechanical Spectroscopy. Trans Tech Publications; 2012. p. 319-24. (Solid State Phenomena; vol. 184).

12. Reina G, Gonzalez-Domınguez JM, Criado A, Vazquez E, Bianco A, Prato M. Synthesis of porous graphene/activated carbon composite with high packing density and large specific surface area for supercapacitor electrode material. AIP Adv [Internet]. Elsevier Ltd; 2017;6(2):290-6. Available from:

10.1016/j.jpowsour.2014.01.056

13. Zheng C, Zhou X, Cao H, Wang G, Liu Z. Synthesis of porous graphene/activated carbon composite with high packing density and large specific surface area for supercapacitor electrode material. J Power Sources [Internet]. Elsevier Ltd; 2014;258:290-6. Available from: http://dx.doi.org/10.1016/j.jpowsour.2014.01.056 14. Politano A, Chiarello G. Probing the Young's modulus and Poisson's ratio in graphene/metal interfaces and graphite: a comparative study. Nano Res. 2015;8(6):1847-56.

15. Sakhaee-Pour A. Elastic properties of single-layered graphene sheet. Solid State Commun [Internet]. Elsevier Ltd; 2009;149(1-2):91-5. Available from: http://dx.doi.org/10.1016/j.ssc.2008.09.050

16. Reina G, González-Domínguez JM, Criado A, Vázquez E, Bianco A, Prato M. Promises, facts and challenges for graphene in biomedical applications. Chem Soc Rev [Internet]. 2017;46:4400-16. Available from: 10.1039/C7CS00363C

17. Gu M, Liu Y, Chen T, Du F, Zhao X, Xiong C, et al. Is Graphene a Promising Nano-Material for Promoting Surface Modification of Implants or Scaffold Materials in Bone Tissue Engineering? Tissue Eng Part B Rev [Internet]. 2014;20(5):477-91. Available from: 10.1089/ten.teb.2013.0638 
18. Podila R, Moore T, Alexis F, Rao A. Graphene Coatings for Biomedical Implants. J Vis Exp [Internet]. 2013;(73):1-9. Available from:

doi:10.1016/j.compscitech.2007.10.033.

19. Niinomi M. Mechanical properties of biomedical titanium alloys. Mater Sci Eng A243. 1998;243:231-6.

20. Gutwald R, Jaeger R, Lambers FM. Customized mandibular reconstruction plates improve mechanical performance in a mandibular reconstruction model. Comput Methods Biomech Biomed Engin [Internet]. Taylor \& Francis; 2017;20(4):426-35. Available from: http://dx.doi.org/10.1080/10255842.2016.1240788

21. Rangel Goulart D, Takanori Kemmoku D, Noritomi PY, de Moraes M.

Development of a Titanium Plate for Mandibular Angle Fractures with a Bone Defect in the Lower Border: Finite Element Analysis and Mechanical Test. J Oral Maxillofac Res [Internet]. 2015;6(3):1-7. Available from: 10.5037/jomr.2015.6305 22. Atilgan S, Erol B, Yardimeden A, Yaman F, Ucan MC, Gunes N, et al. A three dimensional analysis of reconstruction plates used in different mandibular defects. Biotechnol Biotechnol Equip. 2010;24(2):1893-6.

23. Mahathi N, Azariah E, Ravindran C. Finite element analysis comparison of plate designs in managing fractures involving the mental foramen. Craniomaxillofac Trauma Reconstr. 2013;6(2):93-8.

24. Ajayan PM, Schadler LS, Braun P V. Related Titles from Wiley-VCH Molecular Imprinting From Fundamentals to Applications Multilayer Thin Films Sequential Assembly of Nanocomposite Materials High Temperature Ceramic Matrix Composites Nanotechnology. Weinheim WILEY-VCH Verlag GmbH Co KGaA. 2003;236.

25. Moniruzzaman M, Winey KI, April R V, Re V, Recei M, May V. Polymer 
Nanocomposites Containing Carbon Nanotubes. Macromolecules [Internet]. 2006;39(16):5194-205. Available from: 10.1021/ma060733p

26. Thostenson ET, Li C, Chou TW. Nanocomposites in context. Compos Sci Technol [Internet]. 2005;65(3-4):491-516. Available from:

10.1016/j.compscitech.2004.11.003

27. Raabe D, Alemzadeh K, Harrison AJL, Ireland AJ. The chewing robot: A new biologically-inspired way to evaluate dental restorative materials. Proc 31st AnnuInt Conf IEEE Eng Med Biol Soc Eng Futur Biomed EMBC 2009. 2009;6050-3.

28. Chen G, Schmutz B, Wullschleger M, Pearcy MJ, Schuetz MA. Computational investigations of mechanical failures of internal plate fixation. Proc Inst Mech Eng Part H J Eng Med. 2010;224(1):119-26.

29. Ramanathan T, Liu H, Brinson LC. Functionalized SWNT/polymer nanocomposites for dramatic property improvement. J Polym Sci Part B Polym Phys. 2005;43(17):2269-79.

30. Ramanathan T, Stankovich S, Dikin D, Liu H, Shen H, Nguyen S, et al. Graphitic Nanofillers in PMMA Nanocomposites-An Investigation of Particle Size and Dispersion and Their Influence on Nanocomposite Properties. J Polym Sci Part B Polym Phys [Internet]. 2007;45(15):2097-112. Available from: 10.1002/polb.21187 31. Jindal P, Pande S, Sharma P, Mangla V, Chaudhury A, Patel D, et al. High strain rate behavior of multi-walled carbon nanotubes-polycarbonate composites. Compos Part B Eng [Internet]. 2013 Feb;45(1):417-22. Available from: 10.1016/j.compositesb.2012.06.018

32. Jindal P, Jyoti J, Kumar N. Mechanical characterisation of ABS/MWCNT composites under static and dynamic loading conditions. J Mech Eng Sci. 2016;10(3):2288-99. 
33. Wang Q, Dai J, Li W, Wei Z, Jiang J. The effects of CNT alignment on electrical conductivity and mechanical properties of SWNT/epoxy nanocomposites. Compos Sci Technol. 2008;68(7-8):1644-8. 


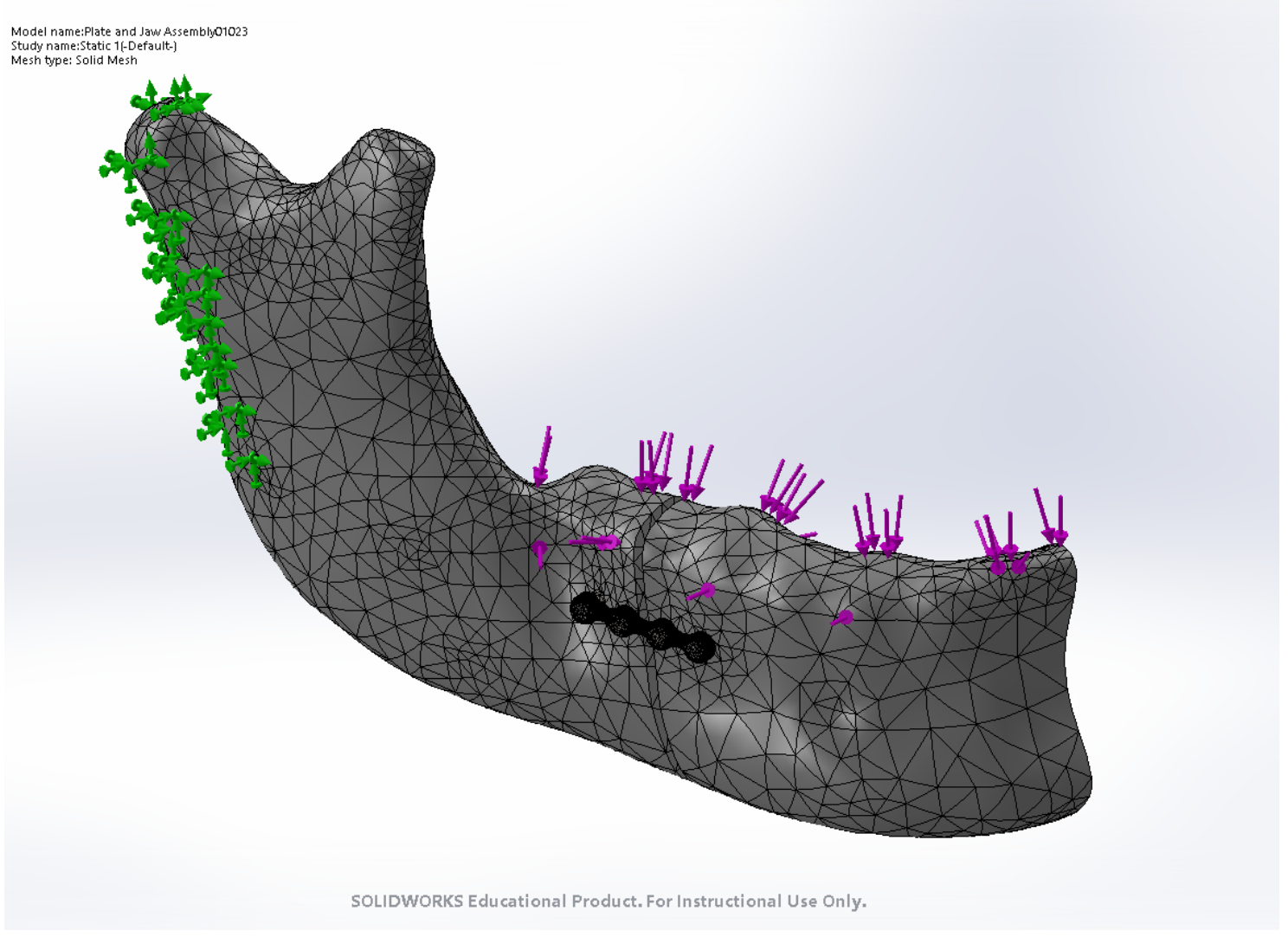

Figure 1. Fractured mandible with a Titanium plate 


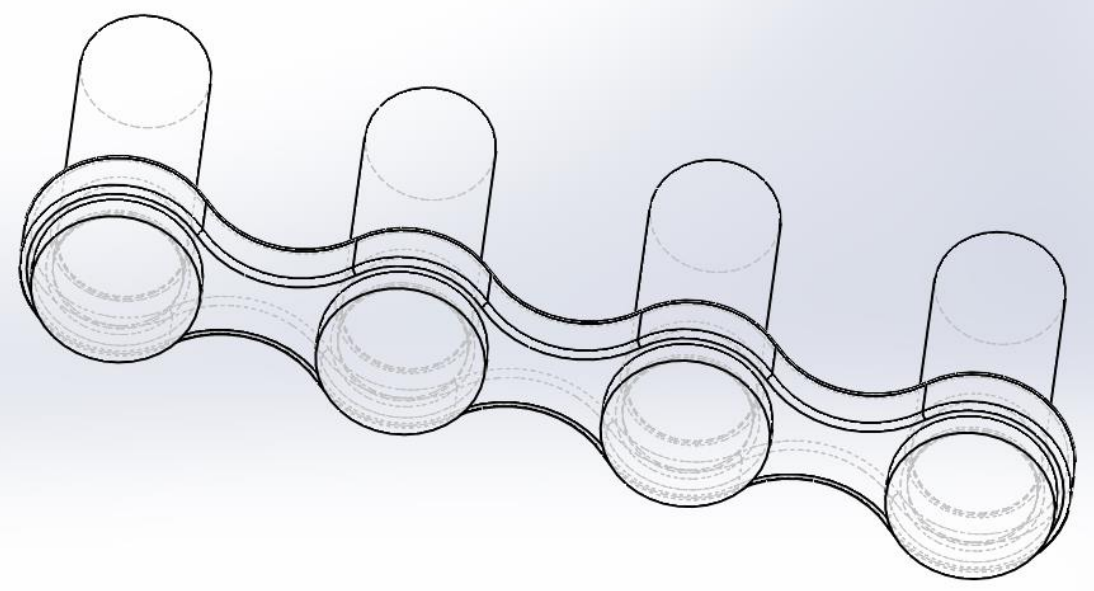

Figure 2. Mandible plate with screws
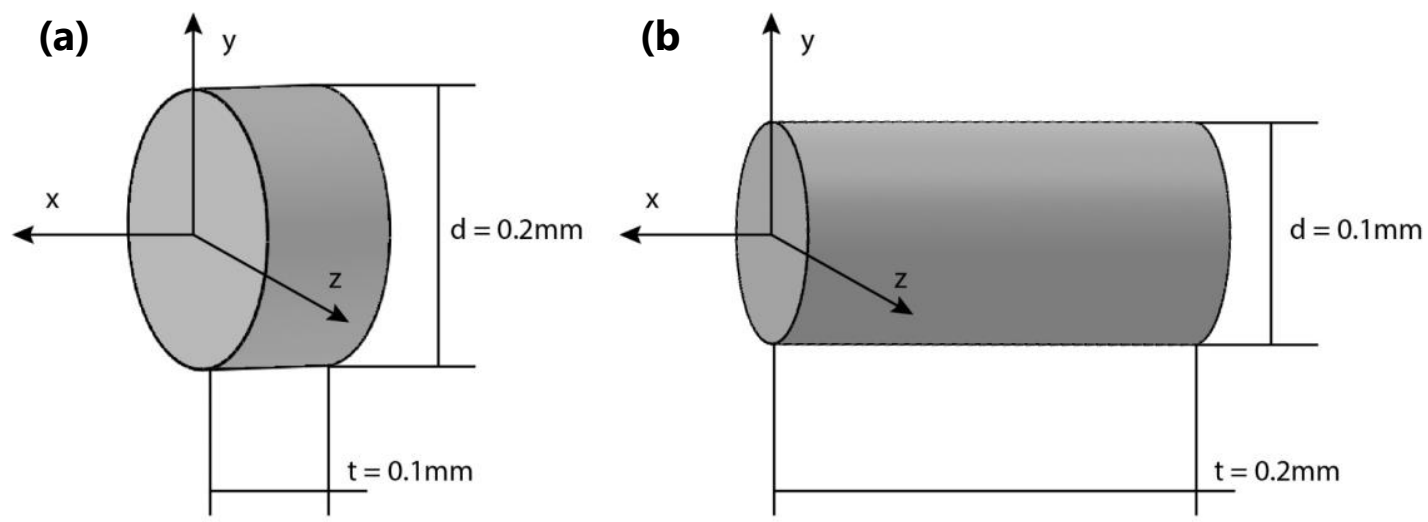

Figure 3 Schematic (a) NP (b) NT 

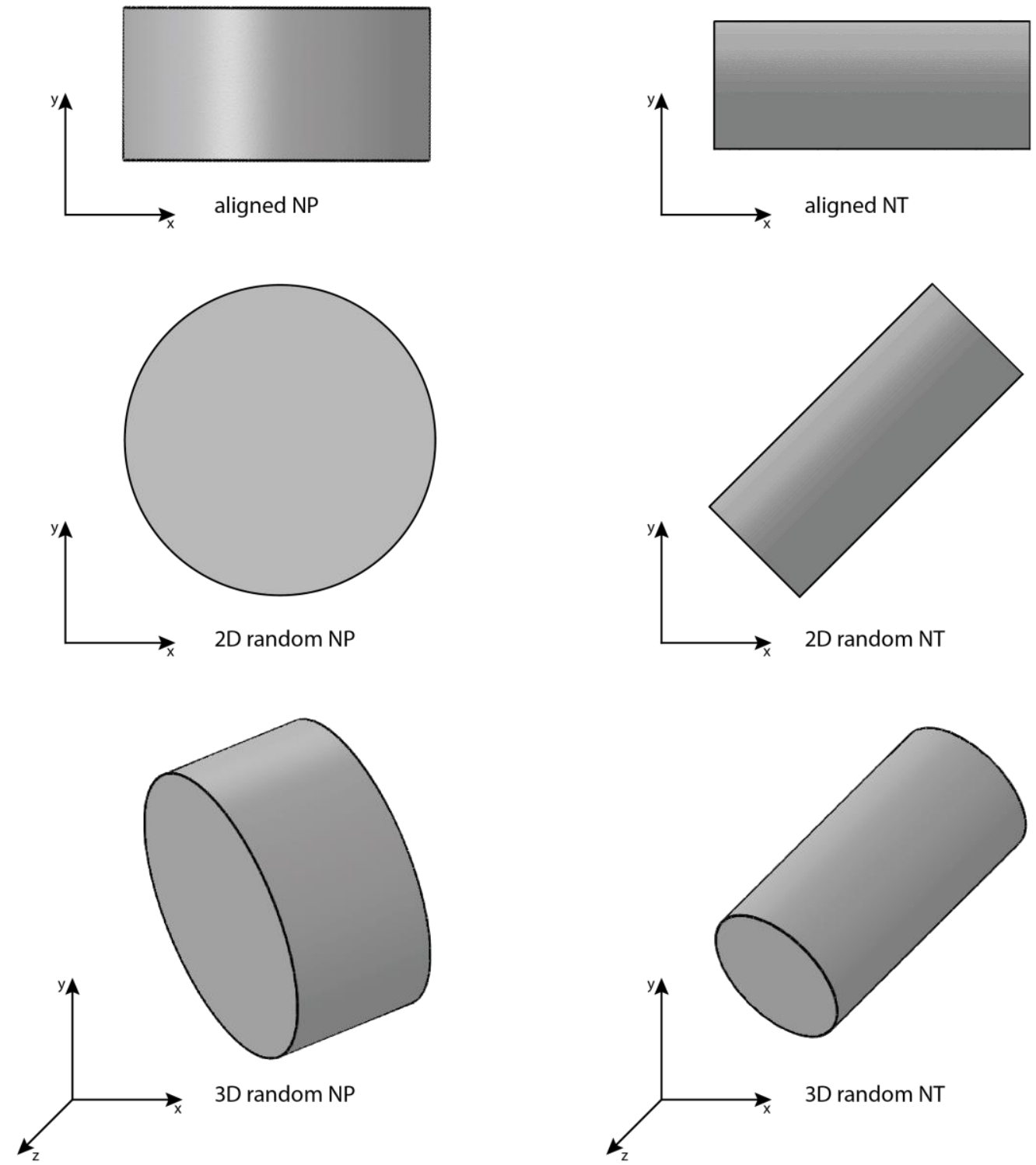

Figure 4 Schematics of nanotube and nanoplatelet nanocomposites with different orientation of nanoparticles: aligned $(\mathrm{y}, \mathrm{x})$; randomly oriented, in-plane $(\mathrm{y}, \mathrm{x})$; and complete 3D randomly oriented (y, $\mathrm{x}, \mathrm{z})$. 


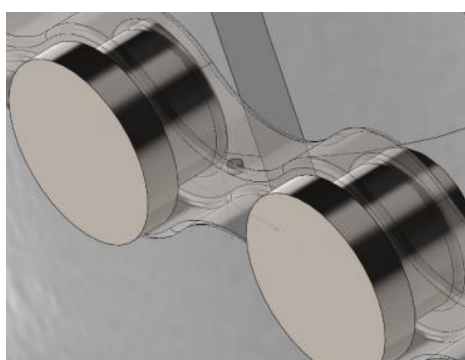

(a) NPs aligned in the plate

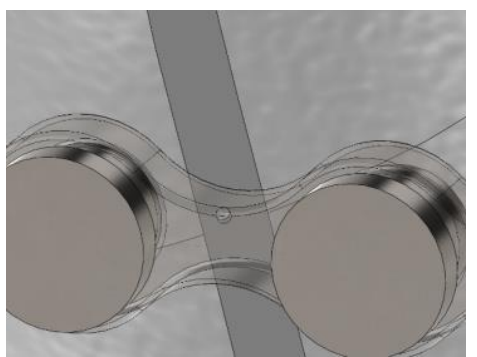

(b) NPs aligned $45^{\circ}$ as $2 \mathrm{D}$ random in the plate

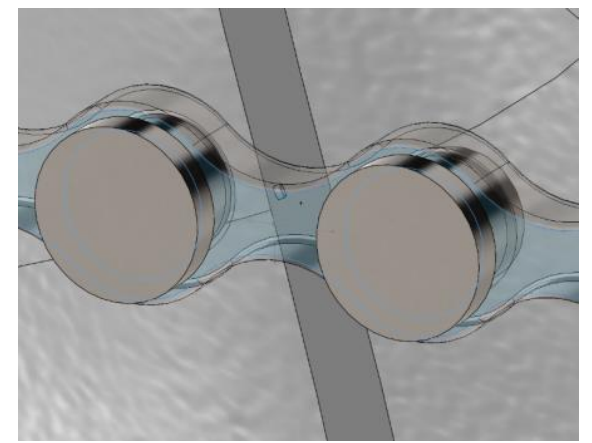

(c) NPs aligned as 3D random inside plate

Figure 5 Different orientations of NPs with plate. 


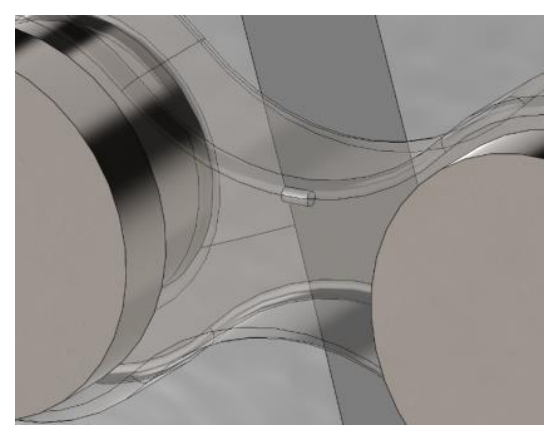

(a) NTs aligned in the plate

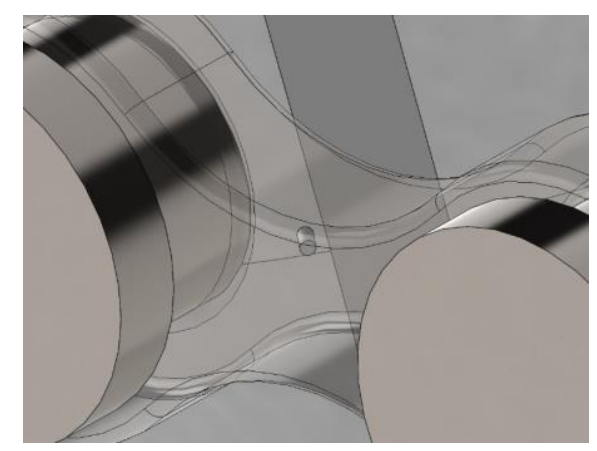

(b) NTs aligned $45^{\circ}$ as 2D random in the plate

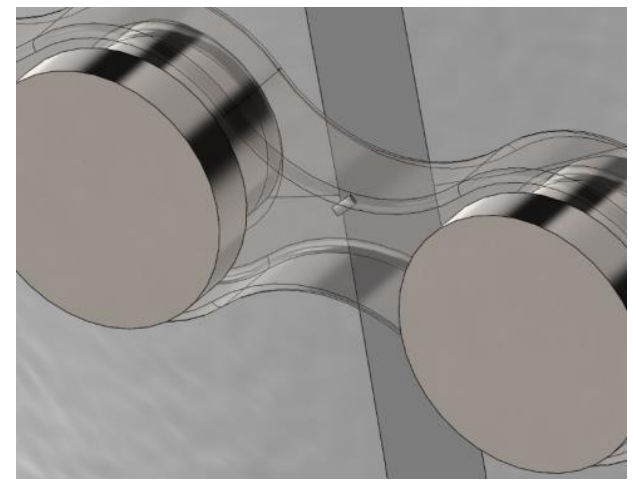

(c) NTs aligned as 3D random inside plate

Figure 6. Different orientations of NTs with plate. 


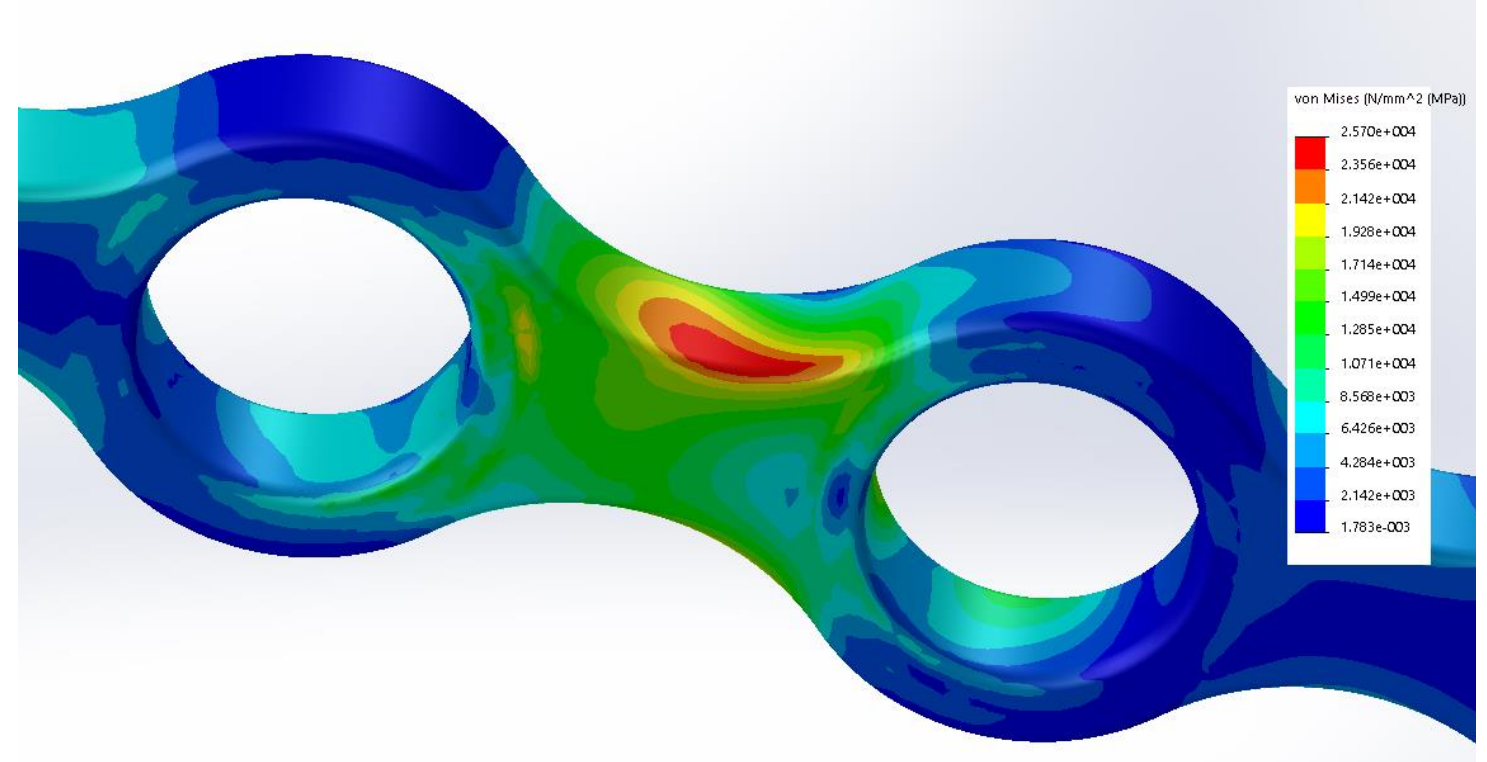

SOLIDWORKS Educational Product. For Instructional Use Only.

Figure 7 (a) Stressed Titanium plate under $500 \mathrm{~N}$ force 


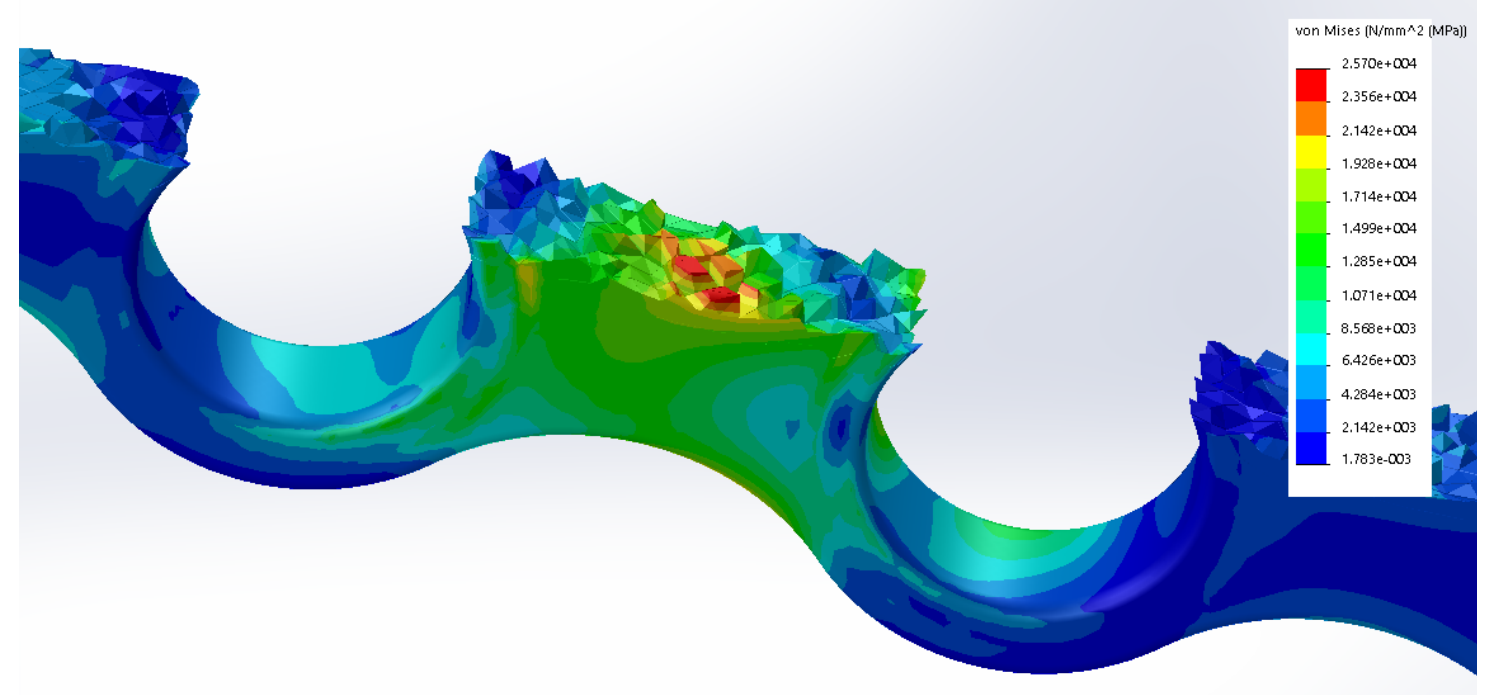

SOLIDWORKS Educational Product. For Instructional Use Only.

Figure 7(b) Sectioned view of the Stressed Titanium plate under 500N force 


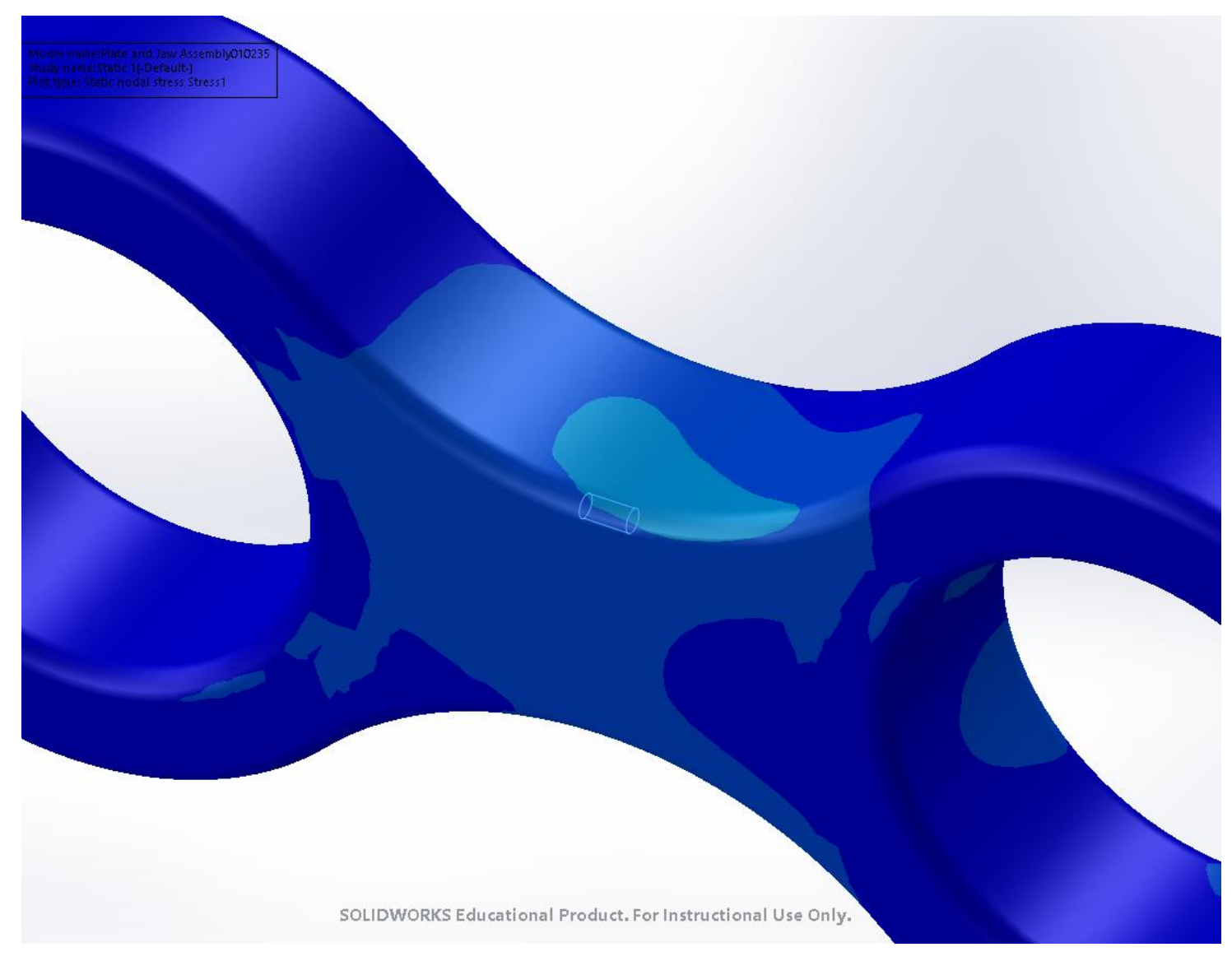

Figure 8(a) Stressed Titanium plate with aligned NTs under 500N force 


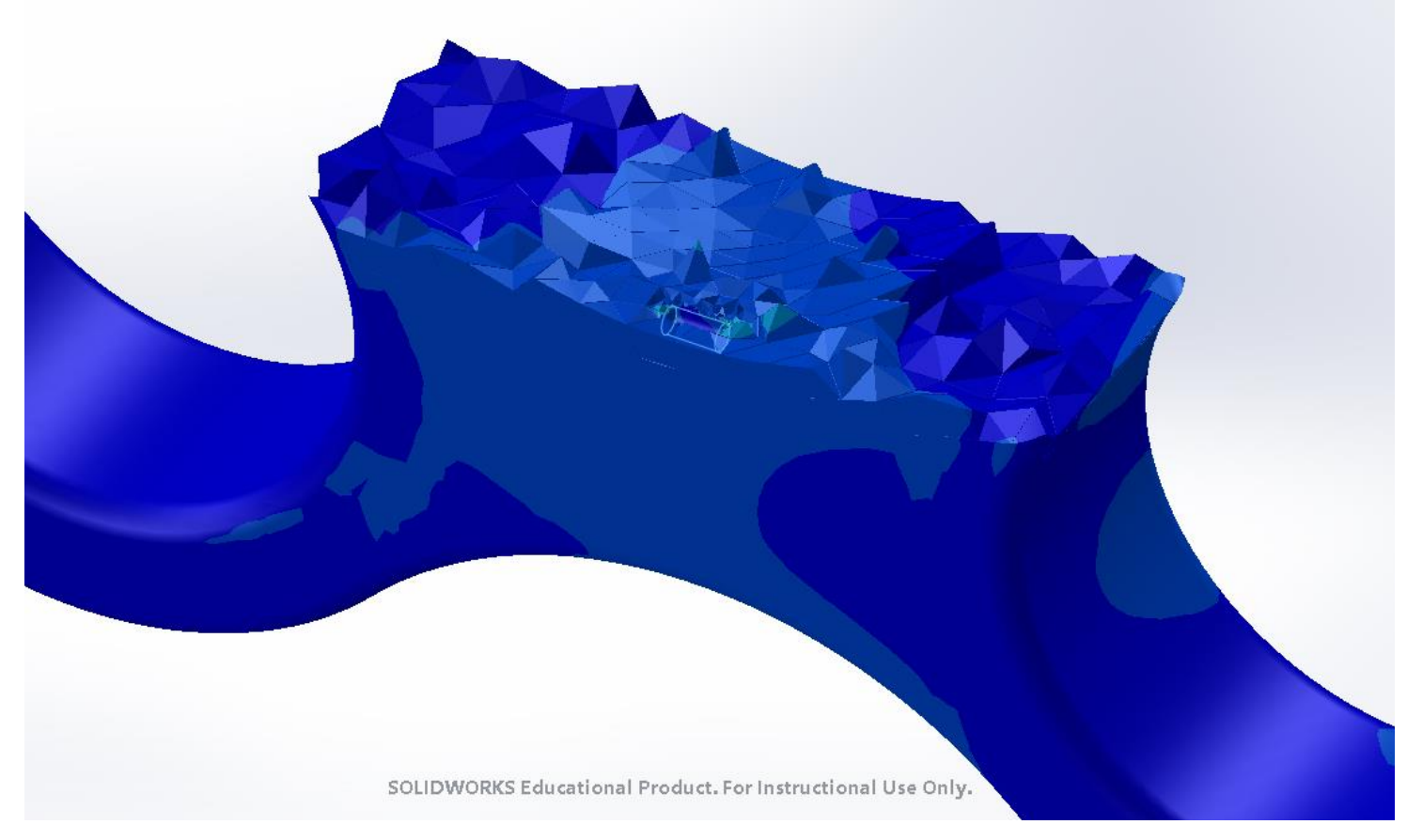

Figure 8 (b) Sectioned view of the Stressed Titanium/aligned NTs under 500N force 


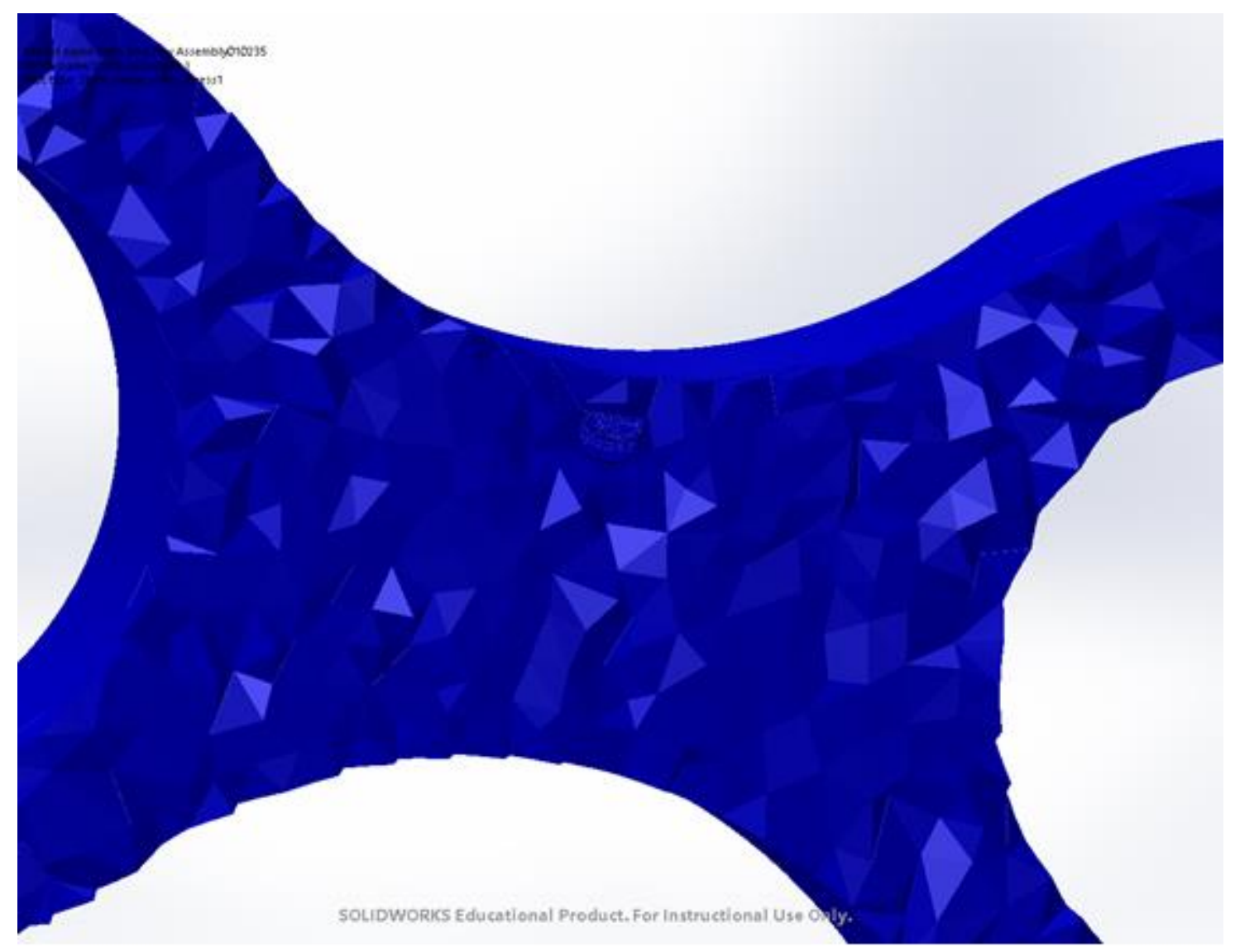

Figure 9 Sectioned view of the Stressed Titanium/2D random NPs under 500N force

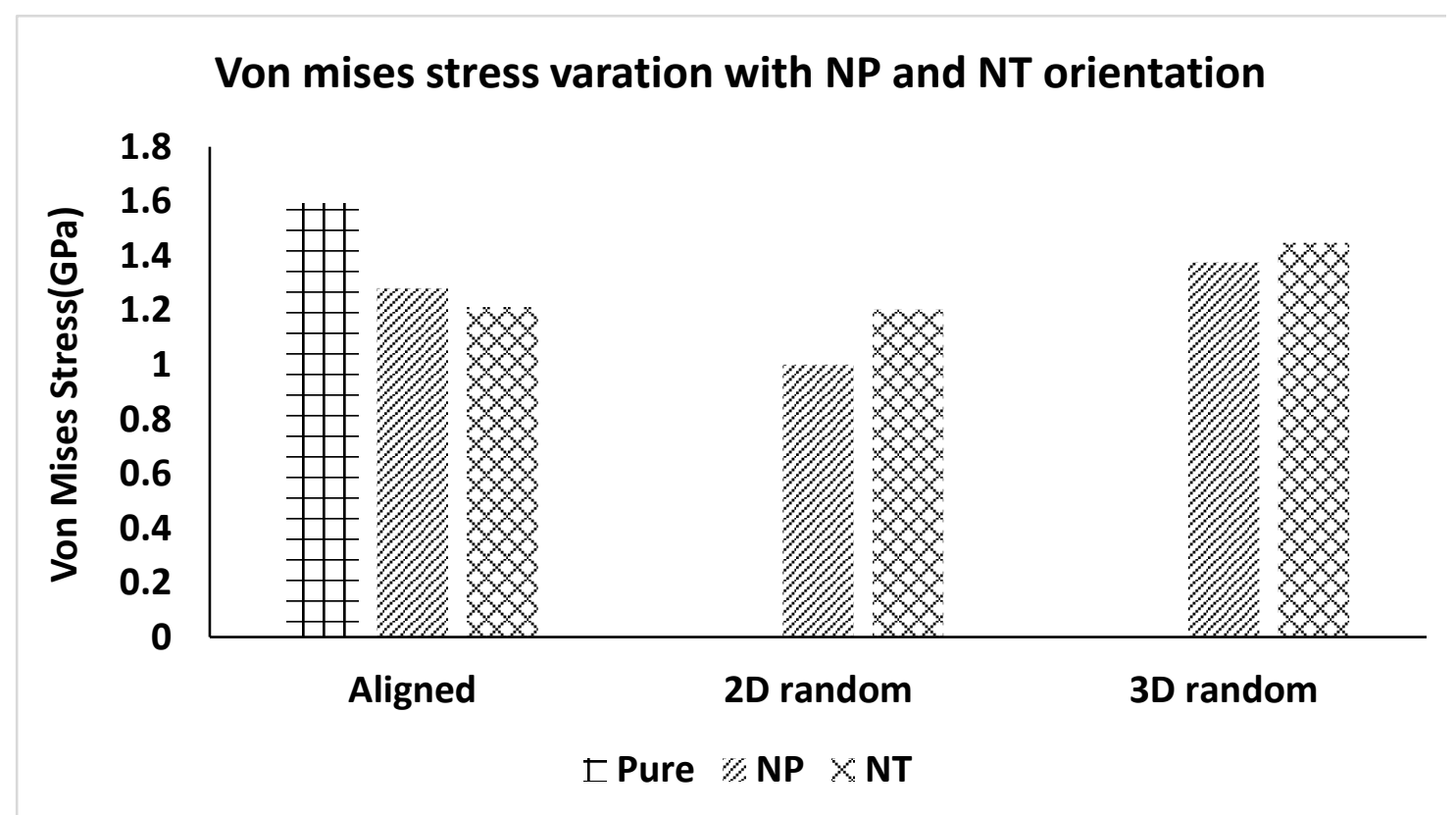


Figure 10 Average Von Mises stresses for pure Titanium alloy plate, NPs and NTs configurations under $500 \mathrm{~N}$ forces

Table 1- Average Von Mises stresses for pure Titanium alloy plate, NPs and NTs configurations under $500 \mathrm{~N}$ forces

\begin{tabular}{|c|l|c|c|}
\hline S.No. & Plate & Average Von Mises stress(GPa) at & Reduction in \\
composition & failure section of plate & stress(\%) \\
\hline 1 & Pure Titanium & 15.91 & \\
\hline 2 & NP aligned & 12.81 & 19.5 \\
\hline 3 & NT aligned & 12.11 & 23.4 \\
\hline 4 & NP 2D random & 10.08 & 13.1 \\
\hline 5 & NT 2D random & 12.03 & 13.6 \\
\hline 6 & NP 3D random & & \\
\hline & NT 3D random & & \\
\hline
\end{tabular}

